\title{
Functional re-recruitment of dysfunctional brain areas predicts language recovery in chronic aphasia
}

\author{
Marcus Meinzer, ${ }^{\mathrm{a}, *}$ Tobias Flaisch, ${ }^{\mathrm{b}}$ Caterina Breitenstein, ${ }^{\mathrm{c}}$ Christian Wienbruch, ${ }^{\mathrm{a}}$ \\ Thomas Elbert, ${ }^{\mathrm{a}}$ and Brigitte Rockstroh ${ }^{\mathrm{a}}$ \\ a Department of Clinical Psychology and Neuropsychology, University of Konstanz, Universitätsstrasse 10, 78457 Konstanz, Germany \\ ${ }^{\mathrm{b}}$ Department of General Psychology, University of Konstanz, Universitätsstrasse 10, 78457 Konstanz, Germany \\ ${ }^{\mathrm{c}}$ Department of Neurology, University of Münster, Albert-Schweitzer-Strasse 33, 48129 Münster, Germany
}

Received 29 May 2007; revised 1 October 2007; accepted 3 October 2007

Available online 18 October 2007

\begin{abstract}
Functional recovery in response to a brain lesion, such as a stroke, can even occur years after the incident and may be accelerated by effective rehabilitation strategies. In eleven chronic aphasia patients, we administered a short-term intensive language training to improve language functions and to induce cortical reorganization under rigorously controlled conditions. Overt naming performance was assessed during functional magnetic resonance imaging (fMRI) prior to and immediately after the language training. Regions of interest (ROIs) for statistical analyses were constituted by areas with individually determined abnormally high densities of slow wave generators (identified by magnetoencephalography prior to the language intervention) that clustered mainly in left perilesional areas. Three additional individually defined regions served to control for the specificity of the results for the selected respective target region: the homologue area of the individual patient's lesion, the mirror image of the delta ROI in the right hemisphere and left hemispheric regions that did not produce a significant amount of slow wave activity.

Treatment-induced changes of fMRI brain activation were highly correlated with improved naming of the trained pictures, but selectively within the pre-training dysfunctional perilesional brain areas. Our results suggest that remodeling of cortical functions is possible even years after a stroke. The behavioral gain seems to be mediated by brain regions that had been partially deprived from input after the initial stroke. We therefore provide first time direct evidence for the importance of treatment-induced functional reintegration of perilesional areas in a heterogeneous sample of chronic aphasia patients. (C) 2007 Published by Elsevier Inc.
\end{abstract}

* Corresponding author. Department of Psychology, University of Konstanz, Universitätsstr.10, P.O. Box 23, 78457 Konstanz, Germany. Fax: +49 7531 882891.

E-mail address: marcus.meinzer@uni-konstanz.de (M. Meinzer).

\section{Introduction}

Aphasia is a common consequence of cerebrovascular stroke and refers to the impaired ability to produce or comprehend language. After a stroke, substantial reorganization of language functions becomes evident and it has been suggested that reactivation of ipsilesional brain areas usually leads to the best outcome during rehabilitation, at least in patients with smaller lesions. Right hemisphere activity might contribute to recovery when substantial language eloquent cortex in the left hemisphere is damaged, as in the case of larger lesions (Heiss and Thiel, 2006; Price and Crinion, 2005). Still, little is known about how treatment impacts on the neural substrates of language in chronic aphasia (usually defined as $>6-12$ months after stroke), and the heterogeneity of lesions and syndromes in aphasia frequently hampers group analyses. To this end, the few studies which examined neuroplastic changes following language intervention by means of functional brain imaging afforded only small and selected samples (for a recent review see Crosson et al., 2007) and yielded controversial results: For example, Leger et al. (2002) reported increased BOLD activity in left perilesional areas in a non-fluent chronic patient after 6 weeks of speech training, while Crosson et al. (2005) demonstrated improved language functions in two chronic non-fluent patients after a treatment of attention that was designed to shift activation from the damaged left to the right hemisphere.

Irrespective of the location of the lesion, cerebrovascular strokes are accompanied by a common marker of dysfunctional information processing in perilesional areas: Focal clusters of slow brain waves in the delta frequency range (1-4 Hz) have been determined from electroencephalography (EEG) and magnetoencephalography (MEG, Lewine and Orrison, 1995). Generators of the slow wave activity are usually located in perilesional areas and are indicative of areas deprived from afferent input (Tecchio et al., 2006). Modulations of pathological slow wave activity have been linked to improved behavioral outcome in a variety of neurological conditions (Lewine et al., 1999; De Jongh et al., 2003; Hensel 
et al., 2004). In 28 chronic aphasics, we (Meinzer et al., 2004) found abnormal MEG slow wave activity to be significantly increased in perilesional areas of the left hemisphere. After 2 weeks of intensive language training slow wave activity had markedly changed (increased or decreased) and the degree of change in either direction was correlated with the improvement in language function. This individually determined functionally abnormal brain area may thus serve as a 'region of interest' in functional imaging analyses when predicting rehabilitation/recovery-related functional changes assessed during a language task and may thus help to clarify the debate about the functional relevance of perilesional areas for treatment-induced language recovery in chronic aphasia. Here, we used functional magnetic resonance imaging (fMRI) and an overt naming task to investigate reorganization before and after a short-term intensive language training in chronic aphasia patients. We hypothesized that, irrespective of site and size of lesion and of aphasic syndrome, more pronounced improvement of language functions after training would be associated with increased task-related brain activation within (perilesional) areas of excessive slow wave activity prior to training.

\section{Materials and methods}

In the present study, a sample of 11 chronic aphasia patients (4 females; age: median 51.0 years, range 19-66; median time since stroke: 32 months, range 6-480) with varying lesion sites and sizes and different aphasia syndromes participated in the study. In all patients, aphasia was caused by a unilateral left hemispheric ischemic or hemorrhagic stroke. All patients were right-handed prior to brain injury, as assessed by the Edinburgh Handedness Inventory (Oldfield, 1971). A chronic state of aphasia was defined as a time since lesion exceeding 6 months. Aphasia was diagnosed according to the guidelines of the standardized German aphasia test battery, the Aachen Aphasia Test (AAT, Huber et al., 1983). Aphasic syndromes were determined as Wernicke's $(N=2)$, Broca's $(N=7)$ and global $(N=1)$ aphasia. One patient could not be categorized according to this schema. Aphasia was classified as mild $(N=4)$, moderate $(N=6)$ or severe $(N=1)$. Patient characteristics are summarized in Table 1. The patients participated in a 10-day intense language training following the CIAT-protocol (Constraint-Induced Aphasia Therapy, Pulvermueller et al., 2001). The development of CIAT has been guided by neuroscientific knowledge about cortical plasticity. It includes a massed training schedule $(3 \mathrm{~h} /$ day for 10 consecutive workdays) within a motivating group setting. This is achieved by way of communicative language games in an interactive group setting. Communication was constrained to spoken language. Improvements in language abilities were systematically shaped by increasing levels of task difficulty adjusted to the individual patient's capacities. During the training period, patients did not participate in any other rehabilitation program (for a detailed description of the procedure see Pulvermueller et al., 2001; Meinzer et al., 2005, 2007a). MEG assessments prior to training and pre- and post-training fMRI scans were obtained from all patients.

Dysfunctional brain areas were defined by focal slow wave activity (obtained from a 5-min MEG session prior to training), while changes in brain activation during an overt picture naming task, determined from fMRI before and after the training, served as indicators of functional changes. Activity changes across the two (pre- and post-training) fMRI scans within areas of individually determined focal abnormal slow wave activity (the delta ROI) were correlated with changes in performance in the naming task. To assess the specificity of the effects for this target ROI, the analyses were repeated for the (a) mirror region of the individual patient's lesion in the right hemisphere (i.e., the homologue area of the lesion), (b) the mirror region of the individually defined delta ROI and (c) the remaining areas of the left hemisphere of each patient that did not produce a significant amount of slow waves prior to training.

Language function outside of the scanner was evaluated by means of the AAT and by a picture naming test that was developed for the purpose of this study. It included 150 photographs of objects (such as a rose, bucket or zebra). Test items were not part of the training materials. Naming of pictures was without response time restriction, the first response being scored. Tests were administered by trained psychologists or speech and language therapists 1 day prior to the onset and 1 day following the completion of the training. Performance on the picture naming tasks during the fMRI scanning and outside of the scanner was evaluated on a 4-point scale in accordance with the scoring system of the AAT naming subtest [3 points: correct response; 2 points: semantic paraphasia (close relationship, e.g., "cigarette" instead of "cigar"), self correction; 1 point: semantic paraphasia (no close relationship - e.g., "bulb" instead of "lighthouse"); 0 point: e.g., fragmentary responses, other articulation].

Table 1

Clinical and demographic parameters of the patient sample

\begin{tabular}{|c|c|c|c|c|c|c|c|c|c|c|}
\hline ID & $\begin{array}{l}\text { Age } \\
\text { (years) }\end{array}$ & Sex & $\begin{array}{l}\text { Onset } \\
\text { (months) }\end{array}$ & Etiology & Syndrome & Severity & $\begin{array}{l}\text { Lesion volume } \\
\left(\mathrm{cm}^{3}\right)\end{array}$ & $\begin{array}{l}\text { Delta ROI } \\
\text { size }\left(\mathrm{cm}^{3}\right)\end{array}$ & $\begin{array}{l}\text { Peak MEG intensity } \\
\text { in delta ROI* }\end{array}$ & $\begin{array}{l}\text { Localization of } \\
\text { maximum DDD }\end{array}$ \\
\hline 1 & 19 & Female & 11 & Hemorrhagic & Broca & Mild & 56.56 & 113.93 & 5.41 & Left mid. frontal \\
\hline 2 & 35 & Male & 32 & Hemorrhagic & Broca & Mild & 212.13 & 82.99 & 5.34 & Left mid. frontal \\
\hline 3 & 49 & Female & 6 & Ischemic & Broca & Mild & 68.92 & 214.93 & 2.79 & Right inf. frontal \\
\hline 4 & 55 & Female & 30 & Ischemic & Not classified & Moderate & 47.40 & 108.19 & 3.76 & Left inf. parietal \\
\hline 5 & 61 & Male & 48 & Ischemic & Broca & Moderate & 66.75 & 95.35 & 5.38 & Left mid. frontal \\
\hline 6 & 60 & Male & 27 & Ischemic & Broca & Moderate & 108.66 & 156.67 & 3.57 & Left mid. frontal \\
\hline 7 & 46 & Male & 34 & Ischemic & Global & Severe & 202.152 & 86.59 & 5.31 & Left inf. frontal \\
\hline 8 & 51 & Male & 59 & Ischemic & Wernicke & Moderate & 121.716 & 76.46 & 3.35 & Left inf. temporal \\
\hline 9 & 56 & Male & 45 & Ischemic & Wernicke & Moderate & 235.956 & 125.42 & 5.09 & Left mid. frontal \\
\hline 10 & 66 & Female & 480 & Ischemic & Broca & Mild & 31.026 & 142.97 & 2.85 & Left sup. temporal \\
\hline 11 & 42 & Male & 19 & Ischemic & Broca & Moderate & 96.84 & 166.17 & 5.38 & Left mid. frontal \\
\hline
\end{tabular}

* $z$-scores above control subjects; mid.=middle, inf.=inferior, sup. $=$ superior; $\mathrm{MEG}=$ magnetoencephalography, DDD= delta dipole density, $\mathrm{ROI}=$ region of interest. 


\section{Data collection}

Written informed consent was obtained from subjects prior to each fMRI and MEG recording. The ethics committee of the University of Konstanz had approved the study protocol and the study was conducted in accordance with the Helsinki Declaration.

A picture naming task during fMRI was chosen because word retrieval is more or less impaired in almost all aphasic patients (Kohn and Goodglass, 1985) and because the language training focused predominantly on naming. The fMRI paradigm employed a temporal sparse sampling design (Hall et al., 1999), in which the overt verbal response is assessed in the scanner during an offphase, and the hemodynamic response is acquired after a short time delay, thereby movement artifacts due to the articulation process are avoided. Magnetic resonance imaging (MRI) was performed using a $1.5 \mathrm{~T}$ scanner (Philips Intera). Functional MRI stimulation consisted of two blocked conditions of overt picture naming, which alternated with passive fixation of a cross at the center of the screen. Conditions (overt naming, passive fixation) were presented in blocks of five consecutive trials, with the sequence of picture naming blocks being identical for both assessments while picture sequence varied within each block. A total of 16 blocks for each condition were collected ( 80 naming trials, 80 fixation trials), high name agreement scores for all stimuli were assured (average name agreement: $0.92 \pm 0.09$ ). Pictures were taken from an Internet database (Szekely et al., 2004) and included line drawings of objects. Forty of these pictures were included in the training material to be named once in each daily training session. An additional 40 untrained objects allowed for the evaluation of a generalization of training effects to untrained pictures. Stimuli were presented by a visor (VisuaStim, Resonance Technology, Inc.) for $3 \mathrm{~s}$, and overt naming was required during this interval. After a delay of $0.8 \mathrm{~s}$ a whole-brain functional MR volume was acquired (temporal sparse sampling; Hall et al., 1999). Prior to the first scan, a training session outside of the scanner was performed to familiarize the patients with the experimental design. A different set of pictures was used for this training session.

Functional MR echo-planar imaging (EPI) was performed with the following acquisition parameters: repetition time $T R=6 \mathrm{~s}$; acquisition time $\mathrm{TA}=2.2 \mathrm{~s} ; 28$ transversal slices, slice thickness: $4.5 \mathrm{~mm}$; in-plane resolution: $3.6 \times 3.6 \mathrm{~mm}$, field of view $=230$, acquisition matrix $64 \times 64$. A total of 166 functional whole-brain volumes were acquired, while the first six scans were excluded from the analysis to allow for T1 equilibration effects. Verbal responses were transmitted from the scanner to a microphone, taperecorded and transcribed after scanning. No overt response was required for the control condition (fixation).

Functional MRI pre-processing was performed using Statistical Parametric Mapping (SPM2, Wellcome Department of Cognitive Neurology, London, UK) and included standard SPM slice-timing, realignment and smoothing $\left(9 \times 9 \times 12 \mathrm{~mm}^{3}\right.$ Gaussian Kernel) routines. During normalization, cost-function masking was performed to prevent distortion of the image due to the lesion (Brett et al., 2001). Data were modeled using a finite impulse response function (Gaab et al., 2007). Anatomic localization of significant voxels within clusters was conducted using the Talairach Demon software (Lancaster et al., 2000) with the nearest gray matter option enabled. Mapping of lesions was performed on a high resolution normalized T1-weighted scan (SPM2 was used for normalization) of each patient using MRIcro (Rorden and Brett, 2000). An average lesion plot was calculated with Matlab (version 6.5) and superimposed on a standard brain template (Montreal Brain).

The magnetoencephalogram (MEG) was recorded prior to the training using a 148-channel whole-head neuromagnetometer (MAGNESTM 2500 WH, 4D Neuroimaging, San Diego, USA) installed in a magnetically shielded chamber. MEG was measured in a supine position for 5 min under resting conditions and the subjects were instructed to fixate a colored mark on the ceiling of the magnetically shielded room throughout the recording in order to avoid eye and head movement. A video camera installed inside the magnetically shielded room allowed for a monitoring of the subject's behavior and compliance throughout the experiment.

The fiducial points, coils and head shape were digitized with a Polhemus 3Space ${ }^{\circledR}$ Fasttrack prior to each measurement. The subject's head position relative to the pickup coils of the sensor was estimated before and after each measurement. MEG was recorded with a sampling rate of $678.17 \mathrm{~Hz}$, using a $0.1-200 \mathrm{~Hz}$ band pass filter. For artifact control, eye movements (EOG) were recorded from four electrodes attached to the left and right outer canthus and above and below the right eye. The electrocardiogram (ECG) was monitored via electrodes attached to the right collarbone and the lowest left rib using a Synamps amplifier $\left(\right.$ NEUROSCAN $\left.{ }^{\circledR}\right)$. Data were reduced by a factor of 16 and digitally filtered for the delta $(1.5-4.0 \mathrm{~Hz})$ frequency band using a digital band pass filter (Butterworth filter of the order 6). Artifact-free time segments were determined by visual inspection. Single equivalent current dipoles were fitted for each time point in the selected artifact free segments (distance of time points $24 \mathrm{~ms}$ ). Five non-overlapping channel groups over left, right, center, anterior and posterior regions were chosen for dipole modeling. A homogeneous sphere, which fits best in a least square sense the digitized patient's head shape below the selected sensors, served as a model for the volume conductor. Dipole fit solutions at time points satisfying the following requirements were accepted: (1) a dipole moment $(q)$ of $10 \mathrm{nAm}<q<100 \mathrm{nAm}$ and (2) a goodness of fit (GOF) greater than 0.90 . These restrictions should ensure that neither artifacts nor small amplitude biological noise would affect the results and that only dipolar fields that were generated by focal sources were analyzed. Each dataset was divided into 1331 voxels, each of $20 \mathrm{~mm}^{3}$, using the AFNI software (Cox, 1996). For each patient, the percentage of dipoles in the delta frequency band per second in each voxel was $z$-transformed and statistically compared to the dipole density distribution of a group of $N=116$ healthy controls who were considered as a 'norm group'. The norm group had been recruited as control subjects in different studies, in which MEG was recorded as described above. Across studies subjects were included as 'control' only, if they had never been treated for a neurological or psychiatric disorder and were not under medication. All subjects were righthanded as verified by the Edinburgh Handedness Inventory. The sample consisted of 59 males, mean age $31.7 \pm 10.9$ years (range 19-66) and 57 females, mean age 26.1 \pm 8.5 years (range 17-64). Since the control group was not matched for age to the patient group it should be mentioned that a recent study in our own laboratory found no differences in the location and extent of slow wave activity in healthy younger and older subjects (Wienbruch, 2007).

For the patient group the individual head frame coordinate system, defined by left and right ear canal and the nasion, was used to transfer and display dipole densities onto an average template brain. Areas with excessive slow wave activity, i.e., high dipole densities in adjacent clusters of voxel, were localized by applying a filter of FWHM $20 \mathrm{~mm}$ (full width half maximum) to the original 
data to minimize the influence of more distant voxels with low dipole density on the areas of higher density. Individual maxima were determined by increasing the threshold until only one voxel appeared to be activated.

Maximum DDD was $>2 z$-scores above that of the control group for all patients (range $2.75-5.83$, for details see Table 1 ) and located in the left hemisphere in $10 / 11$ patients. In one patient only (who displayed the shortest time since stroke) the voxel of maximum slow wave activity was located in the right hemisphere even though also substantial left hemispheric slow wave activity was observed in this patient. Areas with excessive focal slow wave activity (which have been shown to be stable in terms of localization across time in a previous study; Meinzer et al., 2004) were defined as the upper $30 \%$ of individually determined voxels with delta dipole density above those of the control group. This area was considered as search volume for a subsequent fMRI region of interest analysis. The control regions [mirror region of the individual delta ROI in the contralesional hemisphere, homologue areas of the individual patient's lesion in the right hemisphere; remaining regions of the left hemispheric ( $\mathrm{LH})$ that did not produce excessive slow waves (i.e., left hemisphere-delta ROI)] were generated using MRIcro to assess the specificity of the results.

\section{Data analyses}

Changes in the functional activation evoked by picture naming (fMRI) after training were evaluated on an individual basis. In a previous MEG study of our group (Meinzer et al., 2004), improved language function was associated with decreased slow wave activity in the majority of patients. Still, a small subgroup evidenced increased slow wave activity after training which was also correlated with improved language performance. Therefore, in the present study, we aimed to assess whether increased and/or decreased functional MRI activation within the delta ROI or in three control regions was associated with improved naming ability. Increased activation after therapy was defined by the contrast [(Naming-Fix $\left.)_{\text {post }}>(\text { Naming-Fix })_{\text {pre }}\right]$. Decreased activation after therapy compared to the first session (before therapy) was analyzed with the inverse contrast.

In a first step, the individual threshold to be used for each patient was determined by a whole volume analysis that included all trials $(N=80$; trained and untrained pictures). An initial voxel threshold was set to $p<.05$ (family wise error corrected, FWE) with a cluster threshold of $k \geq 10$ voxels. Since we were interested in both increased and decreased fMRI activity, the threshold was lowered to $p<.001$ (uncorrected) when either no increase or decrease of activation was evident at $p<.05$ FWE-corrected (this was the case for patients 2, 3 and 11).

The subsequent ROI analyses were conducted separately for trained $(N=40)$ and untrained $(N=40)$ pictures. Here, the sum of the increased/decreased number of voxels in regions overlapping with the extent of the delta ROI and the three control regions was chosen as the dependent variable during fMRI. The same statistical threshold was chosen as in the previous analyses.

Improvement of language test performance after treatment was scrutinized with one-tailed $t$-tests. Additionally, individual improvement in the AAT was assessed for each patient according to the norms of the AAT for the profile score and all subtests/subscales (critical differences). Differential improvements for trained and untrained items of the fMRI naming task were assessed with repeated measures analysis of variance (ANOVA). Associations between continuous variables (e.g., increased voxel number, percentage increase of naming performance) were assessed with Pearson correlation coefficients.

To assess whether increased activity within the delta ROI was more strongly correlated with improved naming performance compared to either of the three control regions, we employed the significance test for correlated correlations (Meng et al., 1992).

\section{Results}

Prior to the training, severity of aphasia, evaluated by the profile score of the Aachen Aphasia Test (AAT, Huber et al., 1983), was predicted by larger lesion volume as indicated by the individual high resolution structural MRI scan $(r=-.65, p=.029$; see Fig. 1A for an overlay plot of lesion locations of all patients). Lesion size was, however, not significantly correlated with performance on the picture naming task at the first pre-training fMRI assessment $(r=-.35, p=.28)$. MEG-derived excessive slow wave activity, although varying in extension across subjects (see Table 1), clustered in perilesional areas of the left hemisphere (see Fig. 1B). In all patients, peak slow wave activity produced $z$-scores greater than 2 when values of a healthy control group were used for $z$-transformation (Table 1).

After training, aphasia severity had decreased, as indicated by significant increases in the profile score (group average: $t(10)=6.9$, $p<.0001$ ) and the subtests of the Aachen Aphasia Test (all subtests $p<.01$ ). In $8 / 11$ patients, significant improvement of language functions in at least one subtest or subscale of the AAT was substantiated (for a detailed description of the language test results, see Supplementary Table 1).

Similarly, performance in the overt picture naming task had significantly improved from pre- to post-training (group average: $t(10)=9.03, p=.0001)$. Performance on the 40 trained pictures $(t(10)=6.90, p<.0001)$ and the 40 untrained object pictures $(t(10)=3.79, p=.002)$ improved, which points to a generalization of the treatment effect. Still, even though not statistically significant there was a trend towards more pronounced improvement for trained than for untrained pictures (interaction of trained/untrained pictures and time: $F(1,20)=3.38, p=.08)$.

Improvement in the additional naming test that was performed after the fMRI scanning session with 150 untrained items also indicated generalization of the training effect to untrained items (group average: $t(8)=3.88, p=.002$, see Table 2 for details of naming performance inside and outside of the scanner).

\section{Functional imaging analyses}

For the trained pictures highly significant correlations of increased activity in both LH ROIs with improved naming performance were substantiated (delta ROI: $r=.79, p=.003$; LH control ROI: $r=.78, p=.004$ ). However, the correlation for the LH ROI that did not produce excessive slow wave activity was clearly determined by two outliers (patients $7 / 11$; removing the two patients resulted in a non-significant correlation $r=.31, p=.41$ ). The correlation of improved naming performance and increased activity in the delta ROI was not affected when excluding these two outliers $(r=.86, p=.002)$, indicating that reorganization of regions that generate abnormal brain activity, i.e., perilesional areas, may be critical to rehabilitative success for trained materials.

Increased activity in the mirror region of the delta ROI in the contralesional hemisphere was not correlated with therapy success 


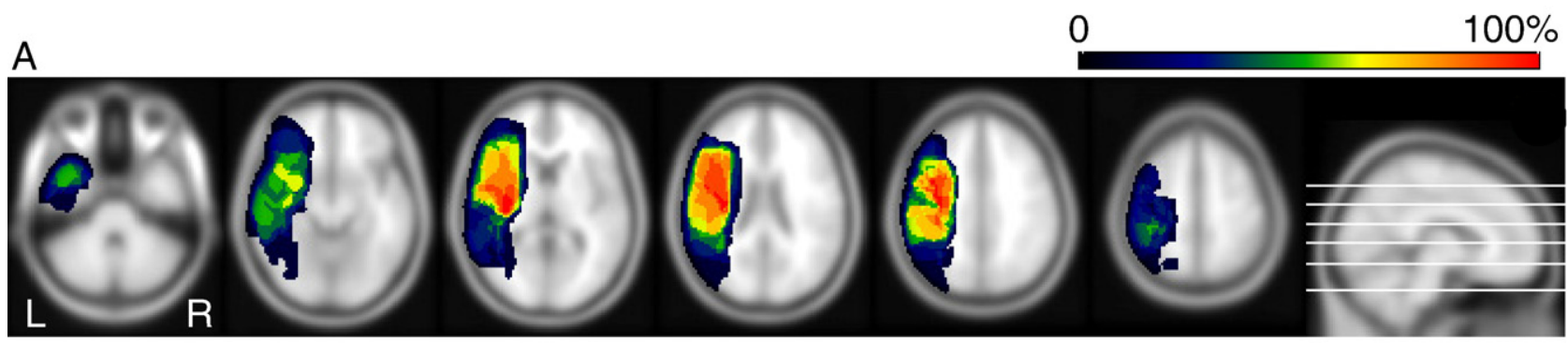

B

0

$100 \%$

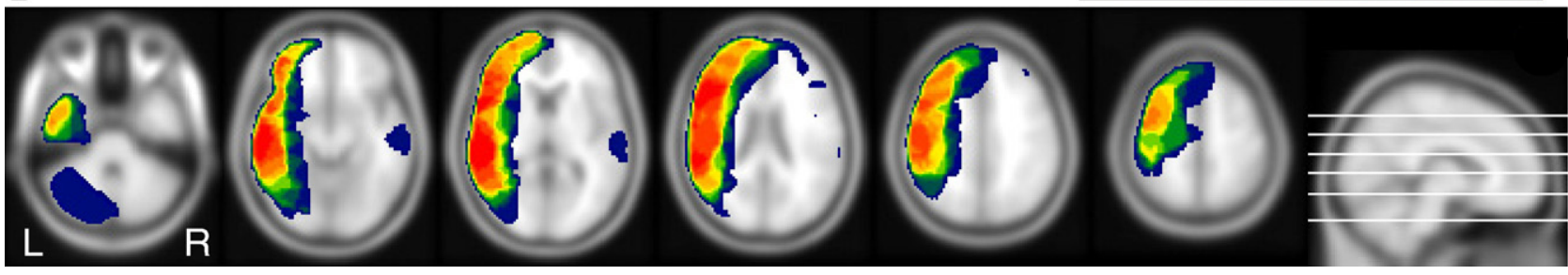

Fig. 1. (A) Lesion overlay plot: lesion size averaged across the eleven patients superimposed onto a standard brain template (ICBM152, Montreal Brain Institute). Yellow and red colors indicate voxels that were more often lesioned across the patient group (left=left). (B) Density of slow wave generators shown here in an overlay plot: areas of excessive slow wave activity (averaged across 11 patients and relative to the distribution of a group of healthy controls, $N=116$ ) superimposed on the same standard brain template. Yellow and red colors indicate voxels with more pronounced slow wave activity across the patient group. Slow wave activity was thresholded (upper $30 \%$ of each patient) to limit the search volume to areas of focal clusters of slow wave activity that have been shown to be stable in location across time, i.e., more than $2 z$-scores above that of the control group (left=left).

(all patients: $r=.06, p=.84$; without patients 7/11: $r=-32, p=.39$ ). A significant correlation between increased activity in the homologue region of the individual patient's lesion in the RH and therapy success was also driven by the two outliers (patients $7 / 11: r=.74$, $p=.008)$. Removing these patients from the analysis resulted in a non-significant correlation $(r=.04, p=.91)$.

Table 2

Naming performance inside and outside of the scanner before (pre) and after (post) the training: naming performance was evaluated on a 4-point scale in accordance with the scoring system of the Aachen Aphasia Test (AAT)

\begin{tabular}{|c|c|c|c|c|c|c|c|c|c|c|c|c|c|c|c|c|c|c|}
\hline \multirow[t]{2}{*}{ ID } & \multicolumn{2}{|c|}{$\begin{array}{l}\text { 3-point } \\
\text { responses } \\
(N)\end{array}$} & \multicolumn{2}{|c|}{$\begin{array}{l}\text { 2-point } \\
\text { responses } \\
(N)\end{array}$} & \multicolumn{2}{|c|}{$\begin{array}{l}1 \text {-point } \\
\text { responses } \\
(N)\end{array}$} & \multicolumn{2}{|c|}{$\begin{array}{l}0 \text {-point } \\
\text { responses } \\
(N)\end{array}$} & \multicolumn{2}{|c|}{$\begin{array}{l}\text { Overall score } \\
\text { fMRI naming }\end{array}$} & \multicolumn{2}{|c|}{$\begin{array}{l}\text { Score } \\
\text { trained } \\
\text { items }\end{array}$} & \multicolumn{2}{|c|}{$\begin{array}{l}\text { Score } \\
\text { untrained } \\
\text { items }\end{array}$} & \multicolumn{2}{|c|}{$\begin{array}{l}\text { Total number of } \\
\text { articulations* }\end{array}$} & \multicolumn{2}{|c|}{$\begin{array}{l}\text { Score external } \\
\text { naming test }\end{array}$} \\
\hline & Pre & Post & Pre & Post & Pre & Post & Pre & Post & Pre & Post & Pre & Post & Pre & Post & Pre & Post & Pre & Post \\
\hline 1 & 21 & 35 & 7 & 2 & 4 & 6 & 5 & 7 & 81 & 116 & 47 & 70 & 34 & 46 & $37(21)$ & $50(27)$ & 254 & 297 \\
\hline 2 & 38 & 54 & 7 & 6 & 11 & 5 & 12 & 2 & 132 & 179 & 58 & 107 & 74 & 72 & $68(34)$ & $67(36)$ & 354 & 368 \\
\hline 3 & 50 & 66 & 12 & 5 & 4 & 4 & 14 & 4 & 178 & 212 & 88 & 112 & 90 & 100 & $80(40)$ & $79(40)$ & 411 & 413 \\
\hline 4 & 25 & 47 & 4 & 4 & 2 & 1 & 35 & 25 & 85 & 150 & 35 & 84 & 50 & 66 & $66(33)$ & 77 (38) & 199 & 229 \\
\hline 5 & 27 & 32 & 11 & 19 & 4 & 4 & 25 & 21 & 104 & 132 & 53 & 73 & 51 & 59 & 67 (33) & $76(38)$ & 234 & 262 \\
\hline 6 & 55 & 69 & 4 & 3 & 3 & 1 & 10 & 4 & 174 & 214 & 90 & 95 & 84 & 119 & 72 (37) & $77(40)$ & 284 & 321 \\
\hline 7 & 6 & 19 & 0 & 2 & 2 & 4 & 50 & 49 & 20 & 65 & 5 & 36 & 15 & 29 & 58 (19) & $74(38)$ & 32 & 56 \\
\hline 8 & 28 & 34 & 5 & 8 & 3 & 0 & 31 & 38 & 97 & 118 & 45 & 62 & 52 & 56 & $67(31)$ & $80(40)$ & 249 & 342 \\
\hline 9 & 20 & 35 & 4 & 3 & 7 & 3 & 13 & 8 & 75 & 117 & 35 & 64 & 40 & 53 & 44 (19) & 49 (24) & 226 & 252 \\
\hline 10 & 39 & 58 & 12 & 8 & 7 & 3 & 12 & 3 & 148 & 193 & 72 & 93 & 76 & 100 & $70(32)$ & $72(33)$ & $\mathrm{n} / \mathrm{a}$ & $\mathrm{n} / \mathrm{a}$ \\
\hline 11 & 17 & 42 & 5 & 8 & 8 & 4 & 35 & 24 & 69 & 146 & 41 & 71 & 28 & 75 & $65(29)$ & $78(40)$ & $\mathrm{n} / \mathrm{a}$ & $\mathrm{n} / \mathrm{a}$ \\
\hline Mean & 29.6 & 44.6 & 6.5 & 6.2 & 5.0 & 3.2 & 22.0 & 16.8 & 105.7 & 149.3 & 51.7 & 78.8 & 54.0 & 70.5 & 63.1 & 70.8 & 249.2 & 282.2 \\
\hline sd & 14.6 & 15.6 & 3.8 & 4.9 & 2.8 & 1.8 & 14.1 & 15.9 & 48.1 & 46.3 & 24.8 & 22.1 & 24.3 & 26.7 & 12.4 & 11.1 & 105.4 & 103.1 \\
\hline $\begin{array}{l}\text { post }>\text { pre } \\
\text { (1-tailed) }\end{array}$ & & & & & $\begin{array}{l}t(10) \\
p<0\end{array}$ & $\begin{array}{l}9.08 \\
01\end{array}$ & $\begin{array}{l}t(10) \\
p<0\end{array}$ & $\begin{array}{l}6.90 \\
01\end{array}$ & $\begin{array}{l}t(10)= \\
p=00\end{array}$ & & $\begin{array}{l}t(10) \\
p=0\end{array}$ & $\begin{array}{l}4.24 \\
08\end{array}$ & $\begin{array}{l}t(8)= \\
p=0\end{array}$ & & & & & \\
\hline
\end{tabular}

Results are given for the overall naming score ( 80 items, maximum score 240 points) as well as for trained and untrained pictures ( 40 each). Additionally, the total number of articulatory speech actions (successful and failed naming attempts that involved articulation) is presented. Results of the external naming test (150 untrained items, maximum score 450) were also scored according to the AAT criteria.

3 points: correct response; 2 points: semantic paraphasia (close relationship, e.g., "cigarette" instead of "cigarre"), self correction; 1 point: semantic paraphasia (no close relationship —e.g., "bulb" instead of "lighthouse"); 0 point: e.g., fragmentary responses, other articulation. n/a: naming outside of the scanner could not be evaluated in two patients due to technical problems with the computer used for stimulus presentation outside of the scanner. * In brackets the total number of articulations for trained pictures before and after training. 
The correlation between improved naming performance and increased activity in the delta ROI was significantly higher compared to the correlation of improved naming with increased activity in the homologue area of the patient's lesions and the mirror image of the delta ROI (lesion homologue: $z=2.11$, $p=.03$; mirror image delta ROI: $z=2.64, p=.008$ ). The post hoc comparison between the two ROIs in the left hemisphere only approached significance $(z=1.84, p=.065)$, even though increased activity in the LH ROI that did not produce a significant amount of slow wave activity per se was not related to improved performance.

Additional analyses confirmed that the perilesional increase of fMRI activation in the delta ROI for trained pictures after training was not related to the size of the individually defined ROIs $(r=$ $-.33, p=.37$ ), indicating that this result was not due to a larger size of the area characterized by excessive slow wave activity in some of the patients. For trained pictures, a lower level of functioning, that is, lower naming performance prior to training, predicted a greater percentage of improvement with training $(r=.73, p=.02)$ and there was a trend for a more pronounced increase of activity in the delta ROI in lower functioning individuals $(r=.57, p=.10)$. Neither post-training improvement on trained pictures nor the respective change in cortical activation was related to duration of aphasia (naming ability: $r=-.22$, $p=.56$, activity inside of the delta ROI: $r=-.29, p=.44)$. Speech activity increased in general (trained items: $t(8)=3.9 p=.002$, Table 2). However, in particular for trained pictures, this increased overall speech activity was not related to increased cortical activity in the delta ROI $(r=.10, p=.78)$. The results were thus specific to lexical performance (see also Table 3 for a summary of regions with increased activity after scanning for trained and untrained pictures in the delta ROI and Supplementary Tables 2 and 3 for increased activity in the two right hemispheric control regions; the simple main effects for picture naming vs. fixation before and after treatment in the delta ROI and in the LH control ROI are listed in Supplementary Tables 4 and 5).

Improvement on untrained pictures was correlated with increased activity in all four ROIs. However, all correlations were driven by the two outliers (patients $7 / 11$; for all ROIs $r>.86$, $p<.001)$. Repeating these analyses without the two patients resulted in a non-significant correlation with performance for all ROIs (delta ROI: $r=-.22, p=.56$; mirror region of the delta ROI in the RH: $r=-.18, p=.64$; homologue area of the lesion in the RH: $r=-.31, p=.40$; remaining LH ROI: $r=-.49, p=.17$ ). Decreased activity for trained and untrained pictures was not correlated with naming performance in any of the ROIs, irrespective of whether we included all patients or excluding the two outliers.

Table 3

Increased activation for trained and untrained pictures after treatment within individual delta ROIs: Increased activation was determined by contrasting naming related activation at the second investigation (post) with the first investigation (pre): [(Naming-Fix $\left.)_{\text {post }}>(\text { Naming-Fix })_{\text {pre }}\right]$

\begin{tabular}{|c|c|c|c|c|c|c|c|c|c|c|c|c|c|c|c|}
\hline \multirow[t]{2}{*}{ ID } & \multirow[t]{2}{*}{ Hemi } & \multirow[t]{2}{*}{ Anatomic structure } & \multicolumn{7}{|c|}{ Trained items } & \multicolumn{6}{|c|}{ Untrained items } \\
\hline & & & BA & $k$ & $T$ & $x$ & $y$ & $z$ & Anatomic structure & BA & $k$ & $T$ & $x$ & $y$ & $z$ \\
\hline 1 & $\mathrm{~L}$ & - & & & & & & & & & & & & & \\
\hline $2^{*}$ & $\mathrm{~L}$ & Middle frontal & $10 / 47$ & 88 & 5.19 & -30 & 62 & 11 & Medial frontal & 32 & 94 & 5.17 & -24 & 41 & 6 \\
\hline $3 *$ & $\mathrm{~L}$ & - & & & & & & & Superior temporal & 22 & 76 & 4.39 & -59 & -37 & 18 \\
\hline \multirow[t]{4}{*}{4} & $\mathrm{~L}$ & Middle frontal & 6 & 203 & 6.86 & -33 & 0 & 53 & Superior frontal & 6 & 102 & 6.37 & -18 & 3 & 61 \\
\hline & & Superior temporal & 22 & 10 & 5.50 & -62 & -17 & 6 & Limbic (uncus) & 36 & 56 & 6.5 & -27 & -2 & 30 \\
\hline & & & & & & & & & Superior temporal & $22 / 42$ & 27 & 5.50 & -56 & -31 & 13 \\
\hline & & & & & & & & & Middle frontal & 6 & 11 & 5.39 & -33 & 14 & 44 \\
\hline \multirow[t]{5}{*}{5} & $\mathrm{~L}$ & Superior temporal & 21 & 39 & 5.85 & -48 & -26 & -4 & Middle occipital & 19 & 94 & 7.57 & -36 & -76 & 4 \\
\hline & & Superior frontal & 10 & 44 & 5.50 & -21 & 59 & 11 & Medial frontal & 8 & 15 & 6.20 & 12 & 37 & 40 \\
\hline & & & & & & & & & Inferior frontal & 44 & 48 & 5.88 & -59 & 7 & 19 \\
\hline & & & & & & & & & Middle temporal & 22 & 16 & 5.67 & -53 & -38 & 5 \\
\hline & & & & & & & & & Middle frontal & 9 & 10 & 5.07 & -45 & 8 & 38 \\
\hline 6 & $\mathrm{~L}$ & Middle frontal & 10 & 40 & 6.53 & -36 & 56 & 8 & Superior temporal & 22 & 15 & 5.66 & -50 & -14 & 3 \\
\hline \multirow[t]{6}{*}{7} & $\mathrm{~L}$ & Middle frontal & 11 & 142 & 8.50 & -21 & 43 & 5 & Anterior cingulate & 10 & 66 & 8.82 & -21 & 44 & -2 \\
\hline & & Transverse gyrus & 41 & 106 & 6.80 & -36 & -26 & 12 & Transverse gyrus & 41 & 178 & 8.15 & -33 & -36 & 13 \\
\hline & & Inferior frontal & 47 & 35 & 6.58 & -30 & 32 & -9 & Inferior frontal & 10 & 37 & 6.18 & -50 & 44 & -2 \\
\hline & & Middle temporal & 22 & 43 & 6.55 & -56 & -35 & 2 & Inferior parietal & 40 & 15 & 5.94 & -48 & -53 & 50 \\
\hline & & Superior frontal & 10 & 11 & 6.08 & -18 & 61 & 0 & & & & & & & \\
\hline & & Inferior temporal & 21 & 26 & 5.60 & -56 & -7 & -17 & & & & & & & \\
\hline 8 & $\mathrm{~L}$ & Insula & 13 & 11 & 5.54 & -36 & -7 & 25 & & & & & & & \\
\hline \multirow[t]{2}{*}{9} & $\mathrm{~L}$ & Fusiform gyrus & $20 / 37$ & 77 & 7.04 & -45 & -12 & -24 & Superior frontal & 10 & 20 & 5.80 & -33 & 56 & 17 \\
\hline & & Middle frontal & 10 & 43 & 6.53 & -36 & 56 & 8 & Fusiform gyrus & 20 & 11 & 5.61 & -39 & -22 & -24 \\
\hline 10 & $\mathrm{~L}$ & - & & & & & & & & & & & & & \\
\hline \multirow[t]{3}{*}{$11^{*}$} & $\mathrm{~L}$ & Middle frontal & 46 & 90 & 4.81 & -45 & 53 & 6 & Inferior temporal & 20 & 1023 & 7.58 & -53 & -30 & -19 \\
\hline & & Superior frontal & 6 & 162 & 4.32 & -12 & -11 & 61 & Superior parietal & 7 & 188 & 5.31 & -39 & -62 & 50 \\
\hline & & & & & & & & & Medial frontal & 9 & 74 & 4.97 & -18 & 44 & 14 \\
\hline
\end{tabular}

$\mathrm{L}=$ left; Hemi $=$ hemisphere; $\mathrm{BA}=$ Brodmann area; $k=$ cluster extent; $x / y / z=$ Talairach-coordinates, $T$-values for maximally activated voxels within significant clusters voxel threshold $p<.05$ FWE-corrected, $k \geq 10 ;{ }^{*} p$-values were set to .001 uncorrected since whole volume analysis revealed no increased or decreased activation at $p<.05$ family wise error corrected; in patients 1/10 increased activity was only evident in right hemispheric areas (see Supplementary Tables 2 and 3 ) and/or in the control ROI in the LH, Pat. 1: trained pictures: precuneus, $k=11, T=5.28, x / y / z$ : $-12 /-44 / 49$; Pat. 10: trained pictures: medial frontal gyrus, $k=55, T=4.8, x / y / z:-18 / 6 / 52$. 


\section{Discussion}

The present results show that cortical plasticity (a) can produce measurable functional outcomes in the very chronic stage of aphasia, (b) occurs predominantly in perilesional areas with dysfunctional processing/deafferentation, and (c) is specifically reflected in an improvement of the trained language function. With respect to clinical rehabilitation, these results substantiate the effects of intensive language training that aims to enhance cortical plasticity based on neuroscientific principles. From a methodological perspective, the results suggest that the combination of imaging techniques, the individual MEG-based definition of dysfunctional perilesional areas and the fMRI-based measurement of functional areas (in an activating task pre- and post-training) allows the study of reorganization/recovery processes even in a sample of patients with different aphasia syndromes, severity of disorder and extension and location of lesion.

The results of the present study suggest the following conclusions:

(1) Improvement of language function in chronic aphasia after intensive training is mediated by measurable cortical reorganization: Language improvement for trained materials was significantly related to increased functional activity (fMRI) within (perilesional) areas of excessive slow wave activity, but across the group of patients not related to functional activation in remaining areas of the left hemisphere or even in the two control ROIs in the right hemisphere (i.e., mirror area of the delta ROI, homologue areas of the lesion in the right hemisphere). It might thus be concluded that improvement was mediated by reintegration of, or increased connectivity within, these perilesional areas. However, as the size of the individual ROI of abnormal slow wave activity varied across patients, it might be argued that the probability of finding increased activation within the delta ROI depends on a larger search volume per se. Argument against this possibility though is that no significant correlation between the size of the search volume and the number of active voxels after treatment was found.

Whereas the initial level of naming ability predicted language improvement, the duration of aphasia was not related to functional changes. This argues against the view that major functional rerecruitment of perilesional areas might be restricted to the acute stage after stroke where physiological factors (like reduction of edema, regression from diachisis) are responsible for improved functional behavior. Rather, the present results emphasize the capacity of reintegration of these areas, in particular in patients with severe chronic aphasia, irrespective of duration, where changes in behavior might only be expected following an intensive training (Robey, 1998; Bhogal et al., 2003).

Increased brain activity after treatment was not restricted to perilesional areas but also evident in remaining areas more distant from the lesion in left hemispheric areas (outside of the delta ROI) and also in the undamaged right hemisphere (the lesion homologue and the mirror ROI of the delta focus) in all patients. Still, the increased activity in these areas was not related to improved language functions which points to a less task specific pattern of activity subserving semantic access (including non-language domain specific functions like memory and attentional resources, Murtha et al., 1999), possibly mediated by a spread of perilesional activation to interconnected brain areas of both hemispheres or increased motor activation (Kurland et al., 2004).

Two patients (7/11; with the lowest pre-training naming scores and the most pronounced relative improvement of naming performance) exhibited not only the strongest reactivation of perilesional brain tissue of all patients, but also substantially increased activity in more distant areas of the left and right hemisphere (e.g., see Table 3/Supplementary Tables 2 and 3). It is unlikely that this large increase of activity in these two patients is specific to lexical access, rather it might be related to increased arousal or attention as a consequence of the intensive stimulation with language. Removing these outlier patients from the analyses resulted in a highly significant correlation between improvement of naming ability and increased activity in perilesional areas characterized by excessive slow wave activity for trained materials. In line with these considerations, more pronounced improvement of language functions (together with a trend for more pronounced activity within the individual delta ROI) was found in patients with lower pre-training naming skills. This might be explained by the fact that patients with more severe aphasia in general do have major problems to use language as a means of communication, and therefore, exhibit less functional communication attempts in everyday life and/or even during therapy. In these patients, the intensive stimulation during the 2 weeks of training interval might have stimulated previously neglected brain areas, with a related marked improvement of naming ability. From these results and considerations, we conclude that improved language function following intensive training was mediated by reorganization/ restitution mainly within pre-training dysfunctional perilesional brain areas of the left hemisphere.

(2) In the present study we demonstrated that the amount of perilesional reactivation in the delta ROI was related to improved naming of trained materials (trained pictures) only. No correlation was found for the untrained materials, even though there was also (but less pronounced) improvement for these items. Additionally, we demonstrated generalization of picture naming performance on untrained items assessed outside of the scanner. Little is known about the mechanism that might support generalization of naming ability in aphasia (and only few studies were able to show such generalization). Certain characteristics of the CIAT procedure might have facilitated behavioral generalization. For example, previous studies that demonstrated generalization of treatment effects in patients with aphasia focused on semantic features of items within superordinate categories (Drew and Thompson, 1999) or on semantically related words to induce spreading activation within semantic networks (Boyle and Coehlo, 1995). Moreover, Kiran and Thompson (2003) investigated the influence of semantic complexity on generalization of treatment effects by modifying typicality of category exemplars. In particular, for (trained) atypical exemplars of a category they found generalization of naming ability to intermediate or typical items, but not vice versa. Similarly, the materials used during the 2-week training period included pictures that were semantically related to the test materials and also included atypical exemplars (e.g., category: animals; test item: horse; training item: tapir). Additionally, the patients were encouraged to use cues to facilitate naming performance, were required to paraphrase targets when they could not come up with the correct word or were provided with superordinate categories ("what kind of an animal is this..."). In combination with the highly intensive training schedule, which engages the semantic system on different levels across an extended time period, this might have enhanced lexical semantic representations and retrieval in general. Subsequent studies are required to elucidate the mechanisms that foster generalization of naming performance following CIAT. 
Still, there is an inconsistency between behavioral improvement (for untrained object names in the scanner) and brain activation patterns that needs to be discussed: Behaviorally, we demonstrated generalization of training effects to untrained items, while this has not been the case for the brain activation patterns (i.e., no correlation for increased brain activity and behavioral improvement; different areas were activated for trained and untrained object names). In particular, only in a few patients was there a partial overlap of areas activated for trained and untrained items. Therefore, different brain areas might be responsible for improvement of trained and untrained items, i.e., specific training effects vs. generalization. This definitively deserves further study.

(3) The combination of individual MEG-assessed pathology and repeated fMRI scanning allowed us to investigate reorganization (or processes related to recovery of function) in a heterogeneous group of patients with chronic aphasia, heterogeneous syndromes, severity and site of lesion. The individual definition of regions of interest, based on individually determined pathology, allows us to examine the functional relevance of brain regions surrounding the lesion during recovery. This impact of perilesional regions/recovery might have been underestimated in previous functional imaging studies with groups of aphasic patients because the overlap of lesions with different size and location might have reduced detection power in these areas (Warburton et al., 1999). This is particularly important in studies employing expressive language designs, like word production, which have been shown to be more strongly bound to the left hemisphere than receptive language tasks (Kinsbourne, 1998). The presently defined individual "pathological" regions of interest allow us to overcome these potential confounds and to assess an unselected sample of patients with different lesion characteristics, aphasic syndromes and severity ranges. Further studies should examine whether the importance of perilesional areas in the rehabilitation of chronic aphasia also holds for (a) other language tasks that are less left lateralized in non-brain damaged individuals as the naming task we used and (b) whether other effective treatment approaches produce a similar pattern of reactivation.

(4) The selection of chronic patients controlled for the confounding effects of spontaneous recovery (restricted to the early post-acute stage) or unspecific factors that might be associated with extended treatment intervals. Patients participated in a short-term intensive language training that aimed at improving the language process targeted during the fMRI task. Improvement of language functions - in particular, naming performance - has been demonstrated by several studies that employed the CIAT procedure (Pulvermueller et al., 2001; Maher et al., 2006; Meinzer et al., 2005, 2006, 2007a,b). Moreover, the patients improved on two naming tests and additional improvement across the group and on an individual basis was substantiated in a standardized language test. At least for the AAT retest stability in chronic aphasia has been demonstrated across short time intervals (retest reliability in chronic aphasia $r>.91$ for all AAT subtests; Huber et al., 1983). Therefore, since patients did not receive any rehabilitation in addition to CIAT during these 2 weeks, the change in language function is most likely to be attributed to the language training. Still, even though the long-term stability of improvements following CIAT has been demonstrated in a previous study (Meinzer et al., 2005), one caveat of the present report is the lack of long-term follow-up assessment to demonstrate the stability of the results.

Another shortcoming of the current study is that no control group of untreated patients was examined with the same MEG and
fMRI protocols to control for non-specific effects of task repetition on brain processes. However, stability of performance and brain activation across repeated fMRI scans has been demonstrated in two recent studies testing nonverbal semantic (Kurland et al., 2004) and object naming in chronic aphasics (Fridriksson et al., 2006). Moreover, a single case report (Meinzer et al., 2006), which compared an aphasic patient and three healthy subjects using exactly the same fMRI design as in the present study, found no group changes in activation patterns across time in (untrained) controls, whereas increased activation was associated with improved naming performance in the patient. Finally, the withinsubject correlation between task improvement and increased perilesional activation emphasizes the functional significance of perilesional changes.

In summary, we have provided first-time direct evidence for the importance of perilesional brain areas to the rehabilitation of expressive language functions in a larger group of chronic aphasia patients and across a wide range of aphasic syndromes. Moreover, the present study provides a new method to investigate (reorganizational) brain processes underlying recovery of function, regardless of individual differences in lesion site and severity and evidence that training procedures fostering cortical reorganization improve language functions in chronic aphasia by remodeling cortical networks in perilesional brain areas, which were disconnected from neuronal network communication after the stroke.

\section{Competing interests statement}

All authors declare that they have no competing financial interests.

\section{Acknowledgments}

This work was supported by the Deutsche Forschungsgemeinschaft (DFG, Grant RO 805/11-4) and the Kuratorium Zentrales Nervensystem (ZNS, Grant 2001013). CB was supported by a grant of the Federal Ministry of Education and Research, Germany (BMBF: 01GW0520) and the Volkswagen Foundation (Az. I/80 708). We gratefully acknowledge the support of $U$. Lommen, B. Awiszus and D. Djundja.

\section{Appendix A. Supplementary data}

Supplementary data associated with this article can be found, in the online version, at doi:10.1016/j.neuroimage.2007.10.008.

\section{References}

Bhogal, S.K., Teasell, R., Speechley, M., 2003. Intensity of aphasia therapy: impact on recovery. Stroke 34, 987-993.

Boyle, M., Coehlo, C., 1995. Application of semantic feature analysis as a treatment for aphasic dysnomia. Am. J. Speech Lang. Pathol. 4, 94-98.

Brett, M., Leff, A.P., Rorden, C., Ashburner, J., 2001. Spatial normalization of brain images with focal lesions using cost function masking. NeuroImage 14, 486-500.

Cox, R.C., 1996. AFNI: Software for the analysis and visualisation of functional magnetic resonance neuroimages. Comput. Biomed. Res. 29, $162-173$.

Crosson, B., Moore, A.B., Gopinath, K., White, K.D., Wierenga, C.E., Gaiefsky, M.E., Fabrizio, K.S., Peck, K.K., Soltysik, D., Milsted, C., 
Briggs, R.W., Conway, T.W., Gonzalez Rothi, L.J., 2005. Role of the right and left hemispheres in recovery of function during treatment of intention in aphasia. J. Cogn. Neurosci. 17, 392-406.

Crosson, B., McGregor, K., Gopinath, K.S., Conway, T.W., Benjamin, M., Chang, Y.L., Moore, A.B., Raymer, A.M., Briggs, R.W., Sherod, M.G., Wierenga, C.E., White, K.D., 2007. Functional MRI of language in aphasia: a review of the literature and the methodological challenges. Neuropsychol. Rev. 17, 157-177.

De Jongh, A., Baayen, J.C., de Munck, J.C., Heethaar, R.M., Vandertop, W.P., Stam, C.J., 2003. The influence of brain tumor treatment on pathological delta activity in MEG. NeuroImage 20, 2291-2301.

Drew, R.L., Thompson, C.K., 1999. Model based semantic treatment for naming deficits in aphasia. J. Speech Lang. Hear. Res. 42, 972-990.

Fridriksson, J., Morrow-Odom, L., Moser, D., Fridriksson, A., Baylis, G., 2006. Neural recruitment associated with anomia treatment in aphasia. NeuroImage 32, 1403-1412.

Gaab, N., Gabrieli, J.D., Glover, G.H., 2007. Assessing the influence of scanner background noise on auditory processing: II. An fMRI study comparing auditory processing in the absence and presence of recorded scanner noise using a sparse design. Hum. Brain Mapp 28, $721-732$.

Hall, D.A., Haggard, M.P., Akeroyd, M.A., Palmer, A.R., Summerfield, A.Q., Elliott, M.R., Gurney, E.M., Bowtell, R.W., 1999. "Sparse" temporal sampling in auditory fMRI. Hum. Brain Mapp. 7, 213-223.

Heiss, W.D., Thiel, A., 2006. A proposed regional hierarchy in recovery of post-stroke aphasia. Brain Lang. 98, 118-123.

Hensel, S., Rockstroh, B., Berg, P., Elbert, T., Schoenle, P.W., 2004. Lefthemispheric abnormal EEG activity in relation to impairment and recovery in aphasic patients. Psychophysiology 41, 394-400.

Huber, H., Poek, K., Weniger, D., Willmes, K., 1983. Aachener Aphasie Test. Hogrefe, Göttingen.

Kinsbourne, M., 1998. The right hemisphere and recovery from aphasia. In: Stemmer, B., Whitaker, H.A. (Eds.), Handbook of Neurolinguistics. Academic Press, San Diego, pp. 386-394.

Kiran, S., Thompson, C.K., 2003. The role of semantic complexity in treatment of naming deficits: training semantic categories in fluent aphasia by controlling exemplar typicality. J. Speech Lang. Hear. Res. 46, 608-622.

Kohn, S.E., Goodglass, H., 1985. Picture-naming in aphasia. Brain Lang. 24, 266-283.

Kurland, J., Naeser, M.A., Baker, E.H., Doron, K., Martin, P.I., Seekins, H.E., Bogdan, A., Renshaw, P., Yurgelun-Todd, D., 2004. Test-retest reliability of fMRI during nonverbal semantic decisions in moderatesevere nonfluent aphasia patients. Behav. Neurol. 15, 87-97.

Lancaster, J.L., Woldorff, M.G., Parsons, L.M., Liotti, M., Freitas, C.S., Rainey, L., Kochunov, P.V., Nickerson, D., Mikiten, S.A., Fox, P.T., 2000. Automated Talairach atlas labels for functional brain mapping. Hum. Brain Mapp. 10, 120-131.

Leger, A., Demonet, J.F., Ruff, S., Aithamon, B., Touyeras, B., Puel, M., Boulanouar, K., Cardebat, D., 2002. Neural substrates of spoken language rehabilitation in an aphasic patient: an fMRI study. NeuroImage 17, 174-183.

Lewine, J.D., Orrison, W.W., 1995. Magnetoencephalography and magnetic source imaging. In: Orrison, W.W., Lewine, J.D., Sanders, J.A.,
Hartshorne, M.F. (Eds.), Functional Brain Imaging. Mosby-Year Book, St-Louis, pp. 404-413.

Lewine, J.D., Davis, J.T., Sloan, J.H., Kodituwakku, P.W., Orrison Jr., W.W., 1999. Neuromagnetic assessment of pathophysiologic brain activity induced by minor head trauma. Am. J. Neuroradiol. 20, 857-866.

Maher, L.M., Kendall, D., Swearengin, J.A., Rodriguez, A., Leon, S.A., Pingel, K., Holland, A., Rothi, L.J., 2006. A pilot study of use-dependent learning in the context of Constraint Induced Language Therapy. J. Int. Neuropsychol. Soc. 12, 843-852.

Meinzer, M., Elbert, T., Wienbruch, C., Djundja, D., Barthel, G., Rockstroh, B., 2004. Intensive language training enhances brain plasticity in chronic aphasia. BMC Biol. 2, 20.

Meinzer, M., Djundja, D., Barthel, G., Elbert, T., Rockstroh, B., 2005. Longterm stability of improved language functions in chronic aphasia after constraint-induced aphasia therapy. Stroke 36, 1462-1466.

Meinzer, M., Flaisch, T., Obleser, J., Assadollahi, R., Djundja, D., Barthel, G., Rockstroh, B., 2006. Brain regions essential for improved lexical access in an aged aphasic patient: a case report. BMC Neurol. 6, 28.

Meinzer, M., Streiftau, S., Rockstroh, B., 2007a. Intensive language training in the rehabilitation of chronic aphasia: efficient training by laypersons. J. Int. Neuropsychol. Soc. 13, 846-853.

Meinzer, M., Obleser, J., Flaisch, T., Eulitz, C., Rockstroh, B., 2007b. Recovery from aphasia as a function of language therapy in an early bilingual patient demonstrated by fMRI. Neuropsychologia 45, 1247-1256.

Meng, X.L., Rosenthal, R., Rubin, D.B., 1992. Comparing correlated correlation coefficients. Psychol. Bull. 111, 172-175.

Murtha, S., Chertkow, H., Beauregard, M., Evans, A., 1999. The neural substrate of picture naming. J. Cogn. Neurosci. 11, 399-423.

Oldfield, R.C., 1971. The assessment of handedness: the Edinburgh inventory. Neuropsychologia 9, 97-113.

Price, C.J., Crinion, J., 2005. The latest on functional imaging studies of aphasic stroke. Curr. Opin. Neurol. 18, 429-434.

Pulvermueller, F., Neininger, B., Elbert, T., Mohr, B., Rockstroh, B., Koebbel, P., Taub, E., 2001. Constraint-induced therapy of chronic aphasia after stroke. Stroke 32, 1621-1626.

Robey, R.R., 1998. A meta-analysis of clinical outcomes in the treatment of aphasia. J. Speech Lang. Hear. Res. 41, 172-187.

Rorden, C., Brett, M., 2000. Stereotaxic display of brain lesions. Behav. Neurol. 12, 191-200.

Szekely, A., Jacobsen, T., D’Amico, S., Devescovi, A., Andonova, E., Herron, D., Lu, C.C., Pechmann, T., Pleh, C., Wicha, N., Federmeier, K., Gerdjikova, I., Gutierrez, G., Hung, D., Hsu, J., Iyer, G., Kohnert, K., Mehotcheva, T., Orozco-Figueroa, A., Tzeng, A., Tzeng, O., Arevalo, A., Vargha, A., Butler, A.C., Buffington, R., Bates, E., 2004. A new on-line resource for psycholinguistic studies. J. Mem. Lang. 51, 247-250.

Tecchio, F., Zappasodi, F., Tombini, M., Oliviero, A., Pasqualetti, P., Vernieri, F., Ercolani, M., Pizzella, V., Rossini, P.M., 2006. Brain plasticity in recovery from stroke: an MEG assessment. NeuroImage (32), 1326-1334.

Warburton, E., Price, C.J., Swinburn, K., Wise, R.J., 1999. Mechanisms of recovery from aphasia: evidence from positron emission tomography studies. J. Neurol. Neurosurg. Psychiatry 66, 155-161.

Wienbruch, C., 2007. Abnormal slow wave mapping (ASWAM) - A tool for the investigation of abnormal slow wave activity in the human brain. J. Neurosci. Methods 163, 119-127. 